\title{
Family-Centered Social Cognitive Factors Preventing Primary Tooth Caries in Children Based on Intervention Mapping Approach
}

\author{
Bahareh Kabiri, ${ }^{1}$ Ali Reza Hidarnia $\mathbb{D D}^{2}$ Mehdi Mirzaei Alavijeh, ${ }^{3}$ \\ Mohammad Esmaeel Motlagh, ${ }^{4}$ and Ali Montazeri $\oplus^{5}$ \\ ${ }^{1}$ Health Education and Health Promotion, Faculty of Medical Sciences, Tarbiat Modares University, Tehran, Iran \\ ${ }^{2}$ Professor, Department of Health Education and Health Promotion, Faculty of Medical Sciences, Tarbiat Modares University, \\ Tehran, Iran \\ ${ }^{3}$ Department of Health Education and Health Promotion, School of Health, Kermanshah University of Medical Sciences, \\ Kermanshah, Iran \\ ${ }^{4}$ School of Medicine, Jundishapur University of Medical Sciences, Ahvaz, Iran \\ ${ }^{5}$ Population Health Research Group, Health Metrics Research Center, Iranian Institute for Health Sciences Research, ACECR, \\ Tehran, Iran
}

Correspondence should be addressed to Ali Reza Hidarnia; hidarnia@modares.ac.ir

Received 15 December 2020; Accepted 30 April 2021; Published 19 May 2021

Academic Editor: Vincenzo Iorio Siciliano

Copyright ( 2021 Bahareh Kabiri et al. This is an open access article distributed under the Creative Commons Attribution License, which permits unrestricted use, distribution, and reproduction in any medium, provided the original work is properly cited.

\begin{abstract}
Background. Given the increasing prevalence of primary tooth caries in Iran and the importance of providing evidence- and theorybased family-centered prevention programs, the present study is aimed at determining the family-centered social cognitive factors preventing deciduous tooth caries among children using the intervention mapping protocol. Methods. This cross-sectional study was performed on 240 Iranian mothers in Ilam who were randomly selected to participate in the study. The data were collected using a self-designed questionnaire including items on demographic information and social cognitive constructs (knowledge, attitude, perceived severity, perceived benefits, perceived barriers, practice guidance, perceived self-efficacy, behavior intention, subjective norms, and social norms). The questionnaire was completed by mothers, and the data were analyzed by performing one-way analysis of variance and linear regression. Results. The results obtained from linear regression analysis showed that perceived self-efficacy $(B=0.295, p<0.001)$, perceived barriers $(B=0.084, p<0.028)$, practice guidance $(B=0.774, p<0.001)$, and social norms $(B=0.137, p<0.020)$ accounted for $71 \%$ of the behavioral intention variance and were the most important predictors for preventing primary tooth caries among children. Conclusion. The findings suggest that perceived self-efficacy, perceived barriers, practice guidance, and social norms are essential for developing family-centered programs to prevent primary tooth caries in children.
\end{abstract}

\section{Introduction}

Primary teeth start to grow about 6 months after birth and complete their growth when children are 3-5 years of age so that they can satisfy their nutritional needs $[1,2]$.

Primary teeth form the foundation of the permanent ones and are very susceptible to caries. Therefore, maintain- ing their health is considered a serious matter for children's health because caries in primary teeth is a major risk factor for its occurrence in permanent teeth [3, 4].

Despite being a very preventable infectious disease, dental caries is still one of the most common chronic childhood diseases, strongly influences the health and social and intellectual development of communities by causing problems 
such as pain, difficulty with eating and speech impairments, and imposes exorbitant costs on families and communities [5-7]. Globally, early childhood caries (ECC) prevalence reported in the world varies from 60 to 90 percent with the developed countries having the lowest and the developing countries the intermediate and/or highest prevalence [8]. The American Academy of Pediatrics (AAP) emphasizes that specialist healthcare for identifying effective factors in oral and dental health in all children must begin at the age of 6 months. Therefore, the first year of life is considered the "critical period" for general or oral and dental health $[9,10]$.

Parents are the first among the social forces that influence children's growth and health in their early years of life. They also play an important role in the development of children's healthy habits, continuation of their healthy behaviors, and prevention of diseases [7].

Mothers play a vital role in children's growth and development, spend a considerable amount of time with them, are directly responsible for their oral and dental health, and play an important role in preventing oral and dental diseases in them [11, 12]. Since oral and dental diseases have a relatively high prevalence in Iran and as no studies based on families and mothers have been conducted in this relation in this country, it is necessary to use a framework for determining predictors related to behaviors that prevent dental caries in primary teeth. There are various available models and theories for health education, but among them, the capability of intervention mapping in planning programs for health promotion has been confirmed in different studies. The intervention mapping protocol addresses issues with the problem-solving perspective using the ecological approach. It was first introduced in 1998 by Kay Bartholomew and Professor Guy Parcel from the University of Texas in Houston in the USA and Professor Kok from Maastricht University in Holland in the Journal Health Education \& Behavior. This protocol has 6 steps: Step 1-the Needs assessment; Step 2-the purposes in changing the behavior of the individuals and environmental factors; Step 3-the selection of theory-based intervention methods and selection or preparation of practical strategies; Step 4-the planning of the intervention program; Step 5-the planning of the programs or their adoption, implementation, and sustainability; and Step 6-the evaluation plan. This research intended to determine the cognitive and social factors related to prevention of dental caries in children by their mothers using the two steps of needs assessment and the objectives in changing the behavior of individuals and environmental factors in the intervention mapping protocol $[13,14]$.

\section{Materials and Methods}

This cross-sectional study was performed on mothers of children aged 6 months to 1 year in Ilam, Iran. The mothers were selected through the random stratified sampling method.
A total of 240 mothers were selected using the following formula:

$$
\begin{aligned}
& n=\frac{\sigma^{2} * z_{1-(\alpha / 2)}^{2}}{d^{2}}, \\
& n=\frac{(0.5)^{2} *(1.96)^{2}}{(0.2)^{2}} 240 .
\end{aligned}
$$

Samples were selected from mothers visiting all 18 health centers in Ilam in proportion to the population of patients at each health center. The inclusion criteria were having a 6month- to 1-year-old child, having a healthy child, having a health record, and literacy, and the exclusion criteria were pregnancy, mother and child underlying disease, separation, and divorce (because the presence of these factors in the mother may affect the process of child care by the mother).

Data were collected using a self-designed questionnaire. The structure of this questionnaire was designed within the intervention mapping approach [15-20]. The questionnaire's items were then discussed and reviewed by the research team. The questionnaire contained items on demographic information such as child's age (month), mother's age (year), mother's education (illiterate/primary, secondary, and higher), mother's occupation (housewife, unemployed, and employed), spouse's age (year), spouse's education, and spouse's occupation (retired, unemployed, and employed) and items on social cognitive constructs including 13 items for assessing the knowledge (Cronbach's $\alpha=0.75$ ) with score ranging from 0 to 26, for example, "primary teeth caries may result in permanent teeth caries," 5 items for assessing the attitude (Cronbach's $\alpha=0.71$ ) with score ranging from 5 to 25 , for example, "It is important for me that my child have healthy and beautiful teeth," 4 items in the perceived severity domain (Cronbach's $\alpha=0.81$ ) with score ranging from 4 to 20 , for example, "Deciduous teeth caries may result in permanent teeth caries," 4 items in the perceived benefits domain (Cronbach's $\alpha=0.71$ ) with score ranging from 4 to 20, for example, "If my child has healthy primary teeth, he will have healthy permanent tooth," 4 items in the perceived barriers domain (Cronbach's $\alpha=0.76$ ) with score ranging from 4 to 20, for example, "Busy life does not allow me to brush my child's teeth every night," 5 items in the perceived self-efficacy domain (Cronbach's $\alpha=0.78$ ) with score ranging from 5 to 25 , for example, "I can provide healthy snacks for my child," 5 items in the behavior intention domain (Cronbach's $\alpha=0.70$ ) with score ranging from 5 to 25 , for example, "I decided to brush my child's teeth after each meal," 3 items in the practice guidance domain (Cronbach's $\alpha=0.73$ ) with score ranging from 3 to 15 , for example, "My spouse reminds me not to forget my child's brush," 4 items in the social norms domain (Cronbach's $\alpha=0.74$ ) with score ranging from 4 to 20 , for example, "It is important for me what others think and say about my child's oral mouth," and 4 items in the subjective norms domain (Cronbach's $\alpha=0.76$ ) with score ranging from 4 to 20, for example, "My friends concern about their child's oral health." It should be noted that all items were scored on a Likert scale from 1 (strongly disagree) 
to 5 (strongly agree). The instrument validity was determined through content validity ratio and content validity index. To assess the content validity index, the questionnaire was provided to 10 health education and health promotion professionals and pediatric dentists, and the necessary corrections were made) all of them were experts in the field of oral health and had done a lot of research in the field of oral health.(

In addition, the construct validity was determined through the exploratory factor analysis. The data were analyzed by performing linear regression, one-way analysis of variance using the SPSS-21 software.

\section{Results}

All 240 mothers participated in the study. The mean (SD) age of mothers was 31.85 (5.67) years. Moreover, $56.3 \%$ of mothers had a university degree, and $72.9 \%$ were housewives. Table 1 shows the demographic characteristics of the participants.

According to the correlation test, there was a statistically significant relationship between maternal age with knowledge, perceived severity, perceived benefits, and perceived selfefficacy. In addition, there was a significant relationship between the child age and two constructs of behavior intention and practice guidance of mothers, so that with increasing age, the behavior intention of mothers to take care of their children's oral care increased. Table 2 shows this relationship.

Linear regression analysis was performed to examine the variables affecting oral health behaviors among children. As presented in Table 3, Model 6 explained 71\% of the variance for oral health behaviors among children. This model was obtained from combining perceived barriers, perceived selfefficacy, practice guidance, and social norms constructs. In addition, there was no significant difference between preventive behaviors for deciduous teeth decay and underlying variables.

\section{Discussion}

Oral health has to be definitely considered an important part of the human organism and not an isolated segment [21].

The findings showed that the perceived self-efficacy, perceived barriers, practice guidance, and social norms were four major and effective cognitive determinants in preventing deciduous tooth caries in Iranian children. The impact of perceived self-efficacy is a reflection of mothers' perceptions of the ease or difficulty of the intended behavior. According to Bandura, self-efficacy is the strongest predictor of behavioral change in the individuals [22]. Since one of the most powerful tools to increase self-efficacy is having adequate skills in performing a behavior, thus, changes in selfefficacy may occur following successful and active participation of individuals in the desired behavior. Many studies have confirmed the role of perceived self-efficacy as an influential construct on behavior $[23,24]$. In the field of oral health, several studies have shown that perceived self-efficacy was one of the strongest determinants of behavior $[25,26]$. In a study, Anagnostopoulos et al. reported that perceived self-efficacy and perceived severity were the predictors of the brushing
TABLe 1: Demographic characteristics of the study subjects.

\begin{tabular}{lcc}
\hline & Number $(n=240)$ & Percentage \\
\hline Child gender & 128 & \\
Male & 112 & 53.3 \\
Female & 240 & 46.7 \\
Mother education & 105 & 100 \\
Secondary & 135 & 43.7 \\
Higher & 240 & 56.3 \\
Father education & 27 & 100 \\
Primary & 78 & 11.2 \\
Secondary & 135 & 32.5 \\
Higher & 240 & 56.3 \\
Mother occupation & 175 & 100 \\
Housewife & 46 & 72.9 \\
Employed & 19 & 19.2 \\
Unemployed & 240 & 7.9 \\
Father occupation & 232 & 100 \\
Employed & 5 & 96.7 \\
Unemployed & 3 & 1.2 \\
Retired & & 1.3 \\
\hline
\end{tabular}

behavior frequency [27]. Similarly studies by Padula and Sullivan and Pimsurang et al. showed that perceived self-efficacy and perceived barriers were two strong predictors of oral health behaviors $[28,29]$. However, some studies have emphasized on the role of objective perceived severity as a powerful predictor of oral health behaviors [30]. Other studies have emphasized on the role of attitude, positive social outcomes, and perceived behavior control in the field of oral care. To achieve oral health behaviors, these studies have suggested ways to develop a more positive attitude toward social norms in individuals [31, 32].

Some studies have emphasized on the role of anxiety and concern as important factors that have a negative impact on oral health and have stated that positive social consequences are effective in having good and healthy teeth $[33,34]$.

In studies by Johns, Champion, and Stretcher, perceived barriers were the strongest predictor of behavior, and reducing them was one of the best programs to influence oral self-care [35].

The current showed that mothers who received more support from people such as dentists, family members, and especially spouses had more intention for oral health care of their children. Many other studies on different health behaviors have also shown the role of support and positive external incentives, so that being reminded by relatives, the impact of abstract norms and the effect of influencers on mothers' behavior were important determinants of oral health care [36, 37].

A study showed that $65 \%$ of the changes in brushing behavior were related to the extended theory of planned behavior [38]. In other studies, the attitude, subjective norms, and perceived behavior control predicted $66.2 \%$ and $63 \%$ of the behavior intention variance in the context of oral health; meanwhile, perceived behavior control was more effective 
TABLE 2: The linear regression model for the constructs of knowledge, attitude, severity, benefits, barriers, self-efficacy, practice guidance, behavior intention, subjective norms, and social norms.

\begin{tabular}{|c|c|c|c|c|c|}
\hline & $B$ & SEB & Standardized B & $T$ & $p$ value \\
\hline \multicolumn{6}{|l|}{ Step 1} \\
\hline Knowledge & 0.018 & 0.043 & 0.022 & 0.426 & 0.671 \\
\hline Attitude & 0.098 & 0.069 & 0.078 & 1.434 & 0.153 \\
\hline Severity & 0.061 & 0.052 & 0.059 & 1.178 & 0.240 \\
\hline Benefits & 0.035 & 0.093 & 0.022 & 0.375 & 0.708 \\
\hline Barriers & 0.082 & 0.039 & 0.101 & 2.085 & 0.038 \\
\hline Self-efficacy & 0.234 & 0.066 & 0.230 & 3.538 & 0.001 \\
\hline Practice guidance & 0.745 & 0.088 & 0.466 & 8.461 & 8.461 \\
\hline Social norms & 0.103 & 0.068 & 0.091 & 1.512 & 0.132 \\
\hline Subjective norms & 0.072 & 0.067 & 0.065 & 1.087 & 0.278 \\
\hline \multicolumn{6}{|l|}{ Step 2} \\
\hline Knowledge & 0.017 & 0.043 & 0.021 & 0.396 & 0.692 \\
\hline Attitude & 0.091 & 0.066 & 0.072 & 1.386 & 0.167 \\
\hline Severity & 0.059 & 0.051 & 0.058 & 1.150 & 0.251 \\
\hline Barriers & 0.082 & 0.039 & 0.101 & 0.101 & 0.038 \\
\hline Self-efficacy & 0.227 & 0.063 & 0.223 & 3.588 & 0.001 \\
\hline Practice guidance & 0.743 & 0.088 & 0.465 & 8.472 & 0.001 \\
\hline Social norms & 0.103 & 0.068 & 0.091 & 1.508 & 0.133 \\
\hline Subjective norms & 0.071 & 0.066 & 0.064 & 1.069 & 0.286 \\
\hline \multicolumn{6}{|l|}{ Step 3} \\
\hline Attitude & 0.097 & 0.064 & 0.077 & 1.514 & 0.131 \\
\hline Severity & 0.065 & 0.049 & 0.063 & 1.322 & 0.187 \\
\hline Barriers & 0.082 & 0.039 & 0.101 & 2.093 & 0.037 \\
\hline Self-efficacy & 0.232 & 0.062 & 0.228 & 3.768 & 0.001 \\
\hline Practice guidance & 0.742 & 0.088 & 0.464 & 8.481 & 0.001 \\
\hline Subjective norms & 0.103 & 0.068 & 0.091 & 1.510 & 0.132 \\
\hline Social norms & 0.071 & 0.066 & 0.064 & 1.076 & 0.283 \\
\hline \multicolumn{6}{|l|}{ Step 4} \\
\hline Attitude & 0.091 & 0.064 & 0.072 & 1.425 & 0.155 \\
\hline Severity & 0.068 & 0.049 & 0.066 & 1.382 & 0.168 \\
\hline Barriers & 0.086 & 0.039 & 0.106 & 2.203 & 0.029 \\
\hline Self-efficacy & 0.249 & 0.060 & 0.245 & 4.182 & 0.001 \\
\hline Practice guidance & 0.744 & 0.088 & 0.465 & 8.505 & 0.001 \\
\hline Social norms & 0.141 & 0.058 & 0.124 & 2.411 & 0.017 \\
\hline \multicolumn{6}{|l|}{ Step 5} \\
\hline Attitude & 0.102 & 0.064 & 0.081 & 1.604 & 0.110 \\
\hline Barriers & 0.096 & 0.039 & 0.118 & 2.499 & 0.013 \\
\hline Self-efficacy & 0.269 & 0.058 & 0.264 & 4.646 & 0.001 \\
\hline Practice guidance & 0.752 & 0.088 & 0.470 & 8.593 & 0.001 \\
\hline Social norms & 0.141 & 0.058 & 0.125 & 2.419 & 0.016 \\
\hline \multicolumn{6}{|l|}{ Step $6^{*}$} \\
\hline Barriers & 0.084 & 0.038 & 0.102 & 2.209 & 0.028 \\
\hline Self-efficacy & 0.295 & 0.056 & 0.290 & 5.299 & 0.001 \\
\hline Practice guidance & 0.774 & 0.087 & 0.484 & 8.925 & 0.001 \\
\hline Social norms & 0.137 & 0.059 & 0.121 & 2.336 & 0.020 \\
\hline
\end{tabular}

*Final model: adjusted $R$-squared $=71.131$ and $p<0.001$.
TABLE 3: Correlation coefficient matrix between cognitive and background variables.

\begin{tabular}{lccc}
\hline & Mother age & Father age & Child age \\
\hline Knowledge & $0.18^{* *}$ & & \\
Perceived severity & $0.23^{* *}$ & $0.16^{*}$ & \\
Perceived benefits & $0.22^{* *}$ & $0.17^{*}$ & \\
Perceived self-efficiency & $0.20^{* *}$ & & \\
Behavior intention & & & $0.19^{* *}$ \\
Practice guidance & & $0.12^{*}$ \\
\hline
\end{tabular}

*Significant at 0.05 level. ${ }^{* *}$ Significant at 0.01 level.

than other variables and was the most powerful predictor of behavior intention $[25,39]$. However, we did not find a significant relationship between underlying variables and social cognitive constructs. In some studies, parental education was the most important determinant of children's socioeconomic status, and there was a more inverse relationship between parental education and caries in developed and developing countries [16]. There are other findings that are consistent with the present study and do not support such a relationship $[40,41]$. Nevertheless, family has an essential role in public health and oral health and is a source for acceptance and transmission of health information, such that health behaviors are constructed and established within the family [42]. Therefore, the role of parents should be taken into consideration when planning for oral health behaviors. In addition, educational programs on children's oral health should reinforce the idea that, despite perceived barriers, parents can observe oral health of their children through promoting self-efficacy. In addition, any planning to reduce barriers will help promote health behaviors.

\section{Conclusion}

The findings showed that cognitive factors, especially four determinants of perceived self-efficacy, perceived barriers, practice guidance, and social norms, have an effective role in preventing primary tooth caries in children. Therefore, it seems necessary to consider the cognitive components when designing intervention programs for preventive behaviors.

\section{Data Availability}

Data will be available if needed.

\section{Conflicts of Interest}

The authors declare that they have no conflicts of interest.

\section{Acknowledgments}

This article is a part of a doctoral thesis funded by Tarbiat Modares University, Tehran, with the ethical code IR.MODARES.REC.1398.021. The authors are grateful to the Research Council of Tarbiat Modarres University and all mothers who participated in this study. 


\section{References}

[1] E. M. Ralph, R. A. David, and A. D. Jeffrey, Dentistry for the child and adolescent, Mosby, New York, 8th edition, 2004.

[2] T. M. Parisotto, C. Steiner-Oliveira, C. Duque, R. C. R. Peres, L. K. A. Rodrigues, and M. Nobre-dos-Santos, "Relationship among microbiological composition and presence of dental plaque, sugar exposure, social factors and different stages of early childhood caries," Archives of Oral Biology, vol. 55, no. 5, pp. 365-373, 2010.

[3] N. Tinanoff and S. Reisine, "Update on Early Childhood Caries Since the Surgeon General's Report," Academic Pediatrics, vol. 9, no. 6, pp. 396-403, 2009.

[4] V. Smaïl-Faugeron, A. M. Glenny, F. Courson, P. Durieux, M. Muller-Bolla, and H. Fron Chabouis, "Pulp treatment for extensive decay in primary teeth," Cochrane Database of Systematic Reviews, vol. 5, article CD003220, 2018.

[5] S. Krisdapong, A. Sheiham, and G. Tsakos, "Oral healthrelated quality of life of 12- and 15-year-old Thai children: findings from a national survey," Community Dentistry and Oral Epidemiology, vol. 37, no. 6, pp. 509-517, 2009.

[6] American Academyof Pediatric Dentistry, "Policy on early childhood caries (ECC): classifications, consequences, and preventive strategies," Pediatric Dentistry, vol. 7, pp. 40-43, 2008.

[7] C. J. L. Murray, M. A. Richards, J. N. Newton et al., "UK health performance: findings of the Global Burden of Disease Study 2010," The Lancet, vol. 381, no. 9871, pp. 997-1020, 2013.

[8] R. Naidu, J. Nunn, and M. Forde, "Oral healthcare of preschool children in Trinidad: a qualitative study of parents and caregivers," BMC Oral Health, vol. 12, no. 1, p. 27, 2012.

[9] S. A. Mani, A. A. Aziz, J. John, and N. M. Ismail, "Knowledge, attitude and practice of oral health promoting factors among caretakers of children attending day-care centers in Kubang Kerian, Malaysia: a preliminary study," N Engl Journal of Indian Society of Pedodontics and Preventive Dentistry, vol. 28, no. 2, pp. 78-83, 2010.

[10] M. H. Wong, S. M. Peng, C. P. J. McGrath, and M. Grath, "Association of infant growth with emergence of permanent dentition among 12 year-aged southern Chinese school children," BMC Oral Health, vol. 19, no. 1, p. 47, 2019.

[11] B. S. Suresh, T. L. Ravisankar, and T. R. Chaitra, "Mother's knowledge about preschool child's oral health," Journal of the Indian Society of Pedodontics and Preventive Dentistry, vol. 4, pp. 282-287, 2010.

[12] J. Baginska and E. Rodakowska, "Knowledge and practice of caries prevention in mothersfrom Bialystok, Poland," International Journal of Collaborative Research on Internal Medicine \& Public Health, vol. 4, pp. 256-266, 2012.

[13] S. Kobel, O. Wartha, T. Wirt et al., "Design, implementation, and study protocol of a kindergarten-based health promotion intervention," BioMed Research International, vol. 2017, Article ID 4347675, 9 pages, 2017.

[14] J. Woodall, J. Woodward, K. Witty, and S. McCulloch, "An evaluation of a toothbrushing programme in schools," Health Education, vol. 114, no. 6, pp. 414-434, 2014.

[15] K. Karami, G. Shakerinejad, K. Ahmad Anghali, and B. Kabiri, Effect of education based on health belief model on the alteration of oral health behaviors among primary female students in Ahvaz in 2012, [M.S. thesis], Jondishapoor Medical Sciences University, Ahvas, 2014.
[16] Z. Saied Moallemi, Oral health among Iranian preadolescents: a school-based health education intervention, [Ph.D. thesis], Academic dissertation university of Helsinki, the Institute of Dentistry, Helsinki, 2010.

[17] A. Mehri and M. Morowatisharifabad, "Utilizing the health promotion model to predict oral health behaviors in the students pf Islamic Azad University of Sabzevar," The Journal of Dental Medicine, vol. 1, pp. 81-88, 2008.

[18] A. Ramezankhani, M. Mazaheri, T. Dehdari, and M. Movahedi, "Relationship between health belief model constructs and DMFT among five-grade boy student in the primary school in Dezfool," Journal of Medical Sciences, vol. 2, pp. 221-228, 2011.

[19] M. Solhi, D. Shojaei Zadeh, B. Seraj, and B. Faghih Zadeh, "The application of the health belief model in oral health education," Iranian Journal of Public Health, vol. 4, pp. 114-119, 2010.

[20] M. E. Buglar, K. M. White, and N. G. Robinson, "The role of self-efficacy in dental patients' brushing and flossing: Testing an extended Health Belief Model," Patient Education and Counseling, vol. 78, no. 2, pp. 269-272, 2010.

[21] C. Cafiero, M. Matarasso, G. Marenzi, V. Iorio Siciliano, L. Bellia, and G. Sammartino, "Periodontal care as a fundamental step for an active and healthy ageing," The Scientific World Journal, vol. 2013, Article ID 127905, 7 pages, 2013.

[22] A. Bandura, "Self-efficacy: toward a unifying theory of behavioral change," Psychology Review, vol. 84, no. 2, pp. 191-215, 1977.

[23] G. Godin and G. Kok, "The theory of planned behavior: A Review of its Applications to health-related Behaviors," American Journal of Health Promotion, vol. 11, no. 2, pp. 87-98, 1996.

[24] I. Ajzen, "The theory of planned behavior," Organizational Behavior and Human Decision Processes, vol. 50, no. 2, pp. 179-211, 1991.

[25] Y. A. B. Buunk-Werkhoven, A. Dijkstra, and C. P. van der Schans, "Determinants of oral hygiene behavior: a study based on the theory of planned behavior," Community Dentistry and Oral Epidemiology, vol. 39, no. 3, pp. 250-259, 2011.

[26] J. Rise, A. N. Astrom, and S. Sotton, "Predicting intentions and use of dental floss among adolescents: an application of the theory of planned behaviour," Psychology and Health, vol. 13, no. 2, pp. 223-236, 1998.

[27] F. Anagnostopoulos, H. Buchanan, S. Frousiounioti, D. Niakas, and G. Potamianos, "Self-efficacy and oral hygiene beliefs about Toothbrushing in dental Patients: A Modelguided study," Behavioral Medicine, vol. 37, no. 4, pp. 132139, 2011.

[28] C. A. Padula and M. Sullivan, "Long-term married couples health promotion behaviors: identifying factors that impact decision-making," Gerontological Nursing, vol. 10, pp. 37-48, 2006.

[29] T. Pimsurang, K. Jaranit, S. Paratap, F. Wijitr, and W. Sarigapan, "Causal relationship between health promoting behavior and quality of life in cervical cancer patients undergoing radiotherapy," Tropical Medicine and Public Health, vol. 6, pp. 1568-1574, 2005.

[30] P. Kasmaei, F. Amin Shokravi, A. Hidarnia et al., "Brushing behavior among young adolescents: does perceived severity matter," BMC Public Health, vol. 14, no. 1, pp. 2-6, 2014. 
[31] Y. A. B. Buunk-Werkhoven, A. Dijkstra, P. Bink, S. van Zanten, and C. P. van der Schans, "Determinants and promotion of oral hygiene behaviour in the Caribbean and Nepal," International Dental Journal, vol. 61, no. 5, pp. 267-273, 2011.

[32] Y. A. B. Buunk-Werkhoven, Y. S. Burrekers, A. Jongboer, D. F. M. Quant, and N. W. D. van Maanen-Schakel, "Determinants of oral hygiene behavior in the Dominican Republic," International Dental Journal, vol. 61, no. 6, pp. 328-333, 2011.

[33] Y. A. B. Buunk-Werkhoven, A. Dijkstra, and C. P. van der Schans, "Oral health-quality of life predictors depend on population," Applied Research in Quality of Life, vol. 4, no. 3, pp. 283-293, 2009.

[34] Y. A. B. Buunk-Werkhoven, A. Dijkstra, R. M. H. Schaub, C. P. van der Schans, and M. Spreen, "Oral health related quality of life among imprisoned Dutch forensic psychiatric patients," Journal of Forensic Nursing, vol. 6, no. 3, pp. 137-143, 2010.

[35] M. Sharma and J. A. Romas, Theoritical foundations of Health Education and Health Promotion, Jones \& Bartlett Learning International, London, 2012.

[36] L. K. Wen, M. D. Shepherd, and M. L. Parchman, "Family support, diet, and exercise among older Mexican Americans with diabetics: a test of an extended health belief model," International Journal of Behavioral Medicine, vol. 3, pp. 191-214, 1997.

[37] L. K. Wen, M. D. Shepherd, and M. L. Parchman, "Family support, diet, and exercise among older Mexican Americans with type 2 diabetes," The Diabetes Educator, vol. 30, no. 6, pp. 980993, 2004.

[38] A. Pakpour Hajiagha and M. Saffari, "Applying theory of planned behavior in predicting brushing in high school students in Qazvin," Journal of Dentistry of Dentists Islamic Association, vol. 3, pp. 201-207, 2012.

[39] H. SMM, "Effect of educational interventions based on theory of planned behavior (tpb) in selecting delivery mode among pregnant women referred to rasht health centers," ZUMS Journal, vol. 77, pp. 94-106, 2011.

[40] M. S. Ullah, A. Aleksejuniene, and H. M. Eriksen, "Oral health of 12-year old Bangladeshi children," Acta Odontologica Scandinavica, vol. 60, no. 2, pp. 117-122, 2002.

[41] J. David, N. J. Wang, A. N. Astrom, and S. Kuriakose, "Dental caries and associated factors in 12-year-old schoolchildren in Thiruvananthapuram, Kerala, India," International Journal of Paediatric Dentistry, vol. 15, no. 6, pp. 420-428, 2005.

[42] J. Holland, M. Mauthner, and S. Sharpe, Family matters: communicating health messages in the family. Health Education Authority Family Health Research Reports, Health Education authority, London, 1996. 